Desarrollo sostenible: informe de un grupo de trabajo

El Grupo de Trabajo sobre Población y Consumo, parte de un grupo de 25 expertos creado en 1993 por el Presidente Clinton a raíz de la Cumbre Mundial de Rio de Janeiro de junio de 1992, ha publicado sus recomendaciones en torno a una estrategia estadounidense en pro del desarrollo sostenible. Se entiende por el mismo toda actividad que puede practicarse indefinidamente sin daño a los recursos ambientales, económicos o sociales y sin reducir la oportunidad de futuras generaciones de aprovechar estos recursos y de tener una vida tan plena como la nuestra o incluso mejor.

Según el Grupo de Trabajo, en los Estados Unidos un sinnúmero de factores sociales, económicos, políticos, demográficos y culturales menoscaban la sostenibilidad, pero en último término el problema radica en el rápido crecimiento de la población y en los patrones de consumo de recursos y de acumulación de desechos. Para afrontar el problema no basta con estabilizar la población, dejando inalterados los patrones de consumo y de producción de desperdicios; tampoco basta con cambiar estos últimos sin estabilizar la población. Cada medida es necesaria y ninguna por sí sola es suficiente.

El abandono de las prácticas ambientales nocivas y su reemplazo por prácticas inocuas son pasos necesarios para lograr la sostenibilidad. Ello implica usar más eficientemente los materiales y la energía empleados en la producción de bienes y servicios y convertir los desechos en recursos, aprovechándolos de tal manera que las plantas industriales puedan eliminar casi por completo sus emanaciones tóxicas y funcionar como circuitos cerrados. La sostenibilidad también exige mantener cierta estabilidad poblacional mediante prácticas de planificación familiar. De hecho, consumo y población son problemas inseparables cuyo manejo, aunque desconcertante debido a la extrema complejidad de los fenómenos sociales, económicos y políticos que entran en juego, depende del establecimiento de estrategias a largo plazo. (Population and Consumption Task Force, President's Council on Sustainable Development. Population and Consumption Task Force report. PCSD; 1996.)

\section{Patrones de consumo de drogas desde 1919}

Tres grandes encuestas domiciliarias han permitido estimar las tasas de incidencia del consumo de drogas en los Estados Unidos de América desde 1919 hasta esta década. Los resultados, publicados en un informe reciente, se basan en el estudio de cohortes que han nacido en el presente siglo y arrojan luz sobre los antecedentes históricos de los patrones de consumo actuales.

Después de la Segunda Guerra Mundial se observó una marcada diferencia en el número de drogas consumidas y en la magnitud del consumo. Solo el tabaco y el alcohol fueron consumidos antes de los 35 años de edad por más de $6 \%$ de las personas que nacieron entre 1919 y 1940; en cambio, 10 drogas distintas fueron consumidas antes de esa edad por más de 6\% de las que nacieron entre 1951 y 1955.

En los años sesenta y principios de los setenta aumentó la incidencia del consumo de todas las drogas menos el cigarrillo. Entre fines de los años setenta y comienzos de los noventa, los patrones de incidencia fueron más complejos y mostraron diferencias según la droga y el grupo de edad. Por ejemplo, la incidencia del consumo de alcohol, sustancias inhalables y analgésicos aumentó, pero casi exclusivamente en el grupo de 12 a 17 años. Bajaron las tasas de incidencia del uso de marihuana, cocaína, tabaco, sedantes y estimulantes, pero en el caso de la cocaína solo a partir de 1989.

Las características sociodemográficas de las personas que empiezan a usar drogas no han variado desde principios de siglo. La incidencia del consumo de alcohol, marihuana y drogas alucinógenas siempre ha sido mayor en hombres y personas de raza blanca, aunque los hispanos han preferido la cocaína. No obstante, también se hallaron diferencias entre la incidencia y prevalencia del uso de cada sustancia. Por ejemplo, en áreas metropolitanas grandes la prevalencia del consumo de alcohol durante el año anterior a la encuesta fue mayor que en zonas rurales o ciudades más pequeñas, pero la incidencia del consumo fue menor.

En la segunda mitad de este siglo el riesgo de una mujer de empezar a fumar ha llegado a ser igual que el de un hombre. El número anual de nue- 
vos consumidores de marihuana ha bajado desde mediados de los años setenta, como también la edad promedio en el momento del primer consumo, y tendencias similares se han observado en el caso de la cocaína. Por otra parte el riesgo de empezar a consumir sustancias alucinógenas y sedantes sin fines terapéuticos se ha mantenido estable desde comienzos de los años setenta. Estos datos revelan la necesidad de tener en cuenta las tendencias observadas en la incidencia del consumo de drogas al idear actividades y políticas de control. (Office of Applied Studies. New report shows trends in drug incidence from 1919 to 1992. SAMSHA News. Verano de 1996:26-27.)

\section{Los priones, partículas proteínicas con infectividad}

A principios de 1996 el gobierno británico dio a conocer una posible asociación entre la encefalopatía bovina espongiforme (EBE) y una variante de la enfermedad de Creutzfeld-Jacob, que afecta a los seres humanos. Más tarde, un grupo de expertos de la OMS declaró que no hay una relación directa y clara entre ambos, pero que los casos encontrados en el Reino Unido ofrecen pruebas indirectas que deben investigarse más a fondo. Se trata de dos enfermedades con períodos de incubación muy largos (de 3 a 10 años) y que se caracterizan por degeneración neurológica progresiva. La mortalidad es de $100 \%$ y no se conoce ningún tratamiento.

Se cree que ambas enfermedades son transmitidas por el agente de la encefalopatía espongiforme transmisible y que el brote en el Reino Unido se debió a la adición de carcasas de animales enfermos al alimento del ganado vacuno, práctica común antes de 1989. En cuanto a la naturaleza de dicho agente, se cree que es más pequeño que la mayor parte de los virus y muy resistente al calor, a los rayos ultravioleta, a la radiación ionizante y a los desinfectantes comunes que normalmente inactivan a los virus. El agente no causa una respuesta inflamatoria o inmunitaria detectable ni se ha observado al microscopio. Tampoco se dispone de pruebas para su detección en seres vivos. Por consiguiente, el diagnóstico se basa en el aspecto esponjoso del tejido cerebral en el examen histológico.

Cuando se aplican procedimientos para la desnaturalización de proteínas, se reduce la infectividad, lo cual sugiere que el agente es de naturaleza proteínica. No parece contener ácido desoxirribonucleico, puesto que los procedimientos que destruyen estos ácidos no reducen su infectividad. Consiguientemente, la comunidad científica se encuentra ante un fenómeno nuevo: la transmisión de una enfermedad infecciosa por agentes proteínicos puros, a los que ha denominado priones para diferenciarlos de los otros agentes ya conocidos. En seres humanos, las encefalitis espongiformes causadas por priones son la enfermedad de CreutzfeldJacob, el síndrome de Gerstmann-StrausslesScheinker, el insomnio familiar letal, el kuru y el síndrome de Alpers. (Anónimo. Priones: ¿nuevos agentes infecciosos? Rev Hosp Niños Buenos Aires 1996;38:141.)

\section{Epidemia de tabaquismo: emergencia de salud pública}

La OMS ha terminado de redactar el primer informe general sobre la situación del tabaquismo en el mundo, en el que se comparan indicadores de la magnitud de la epidemia en distintas regiones. Según los resultados, cerca de 1100 millones de personas (una tercera parte de la población mayor de 14 años) fuman con regularidad, y aunque el tabaquismo ha disminuido en países desarrollados desde principios de los años ochenta, la tendencia se ha visto compensada por un aumento comparable en países en desarrollo.

Gracias al estudio de la OMS, ahora se tiene una mejor idea de la magnitud de la epidemia de tabaquismo y con ello se torna factible tomar medidas para contrarrestarla. El informe también contiene información sobre la morbilidad y mortalidad asociadas con el consumo de tabaco en cada región y da la prevalencia estimada del tabaquismo sobre la base de encuestas efectuadas en $85 \%$ de la población mundial. Se observa, por ejemplo, que de los años setenta a los noventa, el consumo de cigarrillos aumentó más en países del Pacífico Occidental que en las otras regiones. En la China, el consumo de tabaco durante ese período aumentó $260 \%$.

El estudio también ha permitido calcular el consumo de cigarrillos en el mundo. Cuando se incluye el bidis, cigarrillo casero muy popular en la India, en el mundo un total de 6,05 $\times 10^{12}$ cigarrillos se fuman cada año. La cifra es tan inmensa que si todos los cigarrillos se colocaran juntos de punta a punta, abarcarían el doble de la distancia entre el sol y la tierra. (World Health Organization. The tobacco epidemic: a global public health emergency. Comunicado de prensa, 23 de mayo de 1996.)

\section{Nuevos cálculos de la mortalidad materna}

Un estudio reciente ha revelado que las cifras tradicionales de mortalidad materna constituyen una grave subestimación. De todos los indicadores 
utilizados para comparar el grado de desarrollo de distintos países, la mortalidad materna es el que mejor refleja disparidades en materia de equidad. Resulta sumamente difícil, sin embargo, estimar la mortalidad materna debido a que pocos países en desarrollo registran los nacimientos y las defunciones o las causas de muerte, siendo menos aún los que anotan la presencia de embarazo en los certificados de defunción.

Desde 1987, la OMS ha estimado la mortalidad materna usando un modelo simple basado en la esperanza de vida de las mujeres. Aunque las estimaciones mundiales y regionales obtenidas de esa forma han sido aceptadas por las autoridades de salud internacionales, la OMS no ha juzgado conveniente dar uso oficial a las estimaciones nacionales obtenidas con ese modelo. En colaboración con el Fondo de las Naciones Unidas para la Infancia (UNICEF), ha ideado una nueva estrategia en que se hacen ajustes que compensan los efectos del subregistro y se usan variables conocidas para derivar la mortalidad materna en países con escasez de datos. Durante el desarrollo del nuevo modelo se pusieron a prueba numerosas variables que influyen en la mortalidad materna, de las cuales las más importantes son la fertilidad total y el acceso a atención de salud durante la gestación. Mientras más nacimientos hay por mujer, mayor es el número de muertes maternas. Por consiguiente, la fertilidad tiene un efecto en el número total de defunciones por causas relacionadas con la maternidad. Una vez que una mujer queda embarazada, el acceso a atención de calidad se convierte en el factor decisivo en lo referente a su salud y supervivencia.

Las nuevas cifras obtenidas mediante esta estrategia son reestimaciones de las anteriores y no reflejan cambios a lo largo del tiempo. Cuantifican el problema en términos exponenciales, sin afán de precisión. De ahí que tanto la OMS como el UNICEF hayan usado razones y cifras redondeadas. Las estimaciones deben servir para orientar acciones adaptadas a las condiciones económicas y sociales que imperen en cada país. Entre ellas es imprescindible, sin embargo, incluir medidas que den mayor acceso a servicios de atención obstétrica de calidad a todas las embarazadas. (World Health Organization. New estimates of maternal mortality. Wkly Epidemiol Rec 1996;71:97-100.)

\section{¿Reducen la fertilidad los enjuagues vaginales?}

Los enjuagues vaginales se han usado desde los comienzos de la civilización. Aunque habitualmente la comunidad médica los recomienda en mujeres con afecciones específicas, nunca ha habido un consenso sobre su utilidad en mujeres sanas. Según una encuesta realizada en Estados Unidos de América en 1988, 37\% de las mujeres entre las edades de 15 y 44 años practican el lavado vaginal. De ellas, alrededor de 18\% lo hacen con frecuencia semanal.

Los enjuagues vaginales pueden producir trastornos, entre ellos infección por clamidias, enfermedad pelviana inflamatoria y embarazo ectópico. Algunos estudios han sugerido una asociación entre el enjuague vaginal y el cáncer de cuello de útero y vagina, y un informe ha revelado un posible menoscabo de la fertilidad. Con el fin de explorar esta última posibilidad más detenidamente, un grupo de investigadores estudió, en una muestra de 840 mujeres casadas que ya habían dado a luz, la probabilidad mensual de quedar embarazada con y sin el uso de enjuagues vaginales.

Las mujeres que practicaban el lavado vaginal mostraron una reducción de $30 \%$ de las posibilidades de quedar embarazadas durante los meses en que trataron de concebir. La relación persistió aun después de hacer ajustes según factores covariantes y no fue posible explicarla en términos del uso de los enjuagues por razones médicas. La reducción tampoco guardó relación con el tipo de sustancia utilizada para hacer los lavados. Las mujeres de 18 a 24 años mostraron una mayor reducción de la fertilidad (50\%) que las mujeres entre los 25 y 29 años (29\%) o entre los 30 y los 39 (6\%).

En conclusión, la práctica del lavado vaginal se asoció con una reducción de la fertilidad y es necesario llevar a cabo otras investigaciones para determinar si se trata de una relación causal. De ser así, hay que determinar hasta qué punto está mediada por el efecto de infecciones pelvianas. En el entretiempo, es necesario advertir a las mujeres que el lavado vaginal puede acarrear consecuencias desfavorables para su salud. (Day Baird D, et al. Vaginal douching and reduced fertility. Am J Public Health 1996;86:844-850).

\section{La terapia hormonal de reemplazo: uso y abandono}

Aunque la terapia hormonal de reemplazo (THR) aporta beneficios bien conocidos, muchas mujeres nunca la inician o la abandonan precozmente. Con objeto de conocer el porcentaje de mujeres que reciben este tratamiento, los resultados terapéuticos que obtienen, el tiempo que siguen el tratamiento y las razones por las que lo abandonan, se efectuó una encuesta de 505 mujeres de 40 a 59 años de edad. Se observó que $43 \%$ de las mujeres 
posmenopáusicas recibían la THR, sin aparente relación entre la prevalencia del uso y la edad, pero sí entre aquella y la escolaridad. Las mujeres en tratamiento declararon sentir menos bochornos y síntomas de depresión y un mejoramiento de la libido y de la capacidad laboral. No obstante, 70 (34,8\%) de las 201 mujeres en tratamiento lo abandonaron después de un período mediano de 6 meses, casi siempre por indicación del médico. Solamente una paciente abandonó la THR debido a la reaparición de sus menstruaciones.

Cuando se aplicaron las tasas de vida, se observó que a los 3 meses de iniciar la THR, seguían en tratamiento 85,6\% de las mujeres; a los 12,69,6\%; y a $\operatorname{los} 48,59,6 \%$. Las mujeres que todavía estaban en tratamiento lo habían estado recibiendo durante un período de alrededor de 20 meses.

Se puede concluir que un buen porcentaje de mujeres reciben THR y que pese a la frecuencia con que lo abandonan en los primeros meses del tratamiento, más de la mitad lo continúan un mínimo de 4 años. Es necesario adoptar medidas para aumentar la adherencia a la THR, meta alcanzable si se mejoran los conocimientos de los médicos al respecto y si se intensifica la enseñanza del tema en las facultades de medicina y en los programas de posgrado. Una posibilidad adicional es la entrega de material escrito a las pacientes que sepan leer para familiarizarlas con los efectos del climaterio en la calidad de su vida y en su riesgo de padecer osteoporosis y trastornos cardiovasculares. Cada tratamiento se deberá iniciar tras un análisis de sus ventajas en cada caso particular. (Blümel JE, et al. Prevalencia y abandono de la terapia hormonal de reemplazo. Rev Chil Obstet Ginecol 1996;61:75-81).

\section{Estudios sobre el uso inadecuado de medicamentos}

La extensa propaganda que rodea a los medicamentos modernos ha llevado a su asimilación en el mundo entero, aun en sociedades poco desarrolladas sin mecanismos de control que garanticen un uso adecuado. El resultado es que las formas en que se distribuyen y consumen estos medicamentos reducen o anulan su eficacia o impiden obtener el efecto deseado. Pero los medicamentos también poseen propiedades psicológicas y sociales que se olvidan cuando se busca racionalizar su uso. Para el médico, marcan el fin de una consulta y cimentan la credibilidad profesional y la lealtad de su clientela. Según algunos estudios, el profesional tiende a recetar para satisfacer las expectativas de los usuarios.

Una revisión reciente de los estudios efectuados sobre el tema permite sacar varias conclusiones. En primer lugar, el problema es ubicuo, pero sus factores determinantes, su magnitud y sus características varían de un lugar a otro, incluso dentro de un mismo país. Por consiguiente, es esencial estudiar los hábitos de prescripción en cada localidad a fin de diseñar intervenciones apropiadas. En segundo lugar, el conocimiento de las buenas prácticas no implica que sean observadas, aun por profesionales bien adiestrados. Cualquier intervención destinada a fomentar las prácticas adecuadas involucra una gran variedad de mensajes y materiales informativos y programas de adiestramiento, siendo estos últimos insuficientes para lograr cambios de conducta sin el refuerzo de intervenciones dirigidas al nivel gerencial. Estas, a su vez, tampoco son fructíferas por sí solas y hasta pueden originar un mercado negro de productos o conductas ilegales. De hecho, algunos autores opinan que tales intervenciones, como la suspensión de un medicamento registrado, meramente provocan la sustitución de un fármaco por otro.

Falta determinar el mejor modo de combinar las diferentes intervenciones para resolver problemas particulares en contextos específicos, pero cualquier logro verdadero dependerá de la capacidad de llegar a todos los grupos que figuran en el ciclo de la medicación: consumidores, proveedores de servicios, distribuidores y fabricantes. Aún no se sabe cómo reaccionarán la industria farmacéutica y la comunidad en general si se reduce la disponibilidad de ciertos fármacos. No obstante, es preciso seguir diseñando mejores intervenciones y poner a prueba teorías específicas sobre cómo cambiar los hábitos de prescripción. (Trostle J. Inappropriate distribution of medicines by professionals in developing countries. Soc Sci Med 1996;42:1117).

\section{El amamantamiento $y$ los motivos de su abandono}

La duración ideal de la lactancia materna siempre ha dado lugar a controversia. En países en desarrollo, dicha duración suele ejercer un impacto muy grande en la mortalidad y morbilidad infantiles y en el estado nutricional de los niños. Cuando se evalúa este impacto, raras veces se incluyen en el modelo de estudio como factores de confusión las razones por las cuales la madre ha decidido terminar el amamantamiento.

La OMS actualmente recomienda que en países en desarrollo la lactancia al pecho se continúe hasta casi finales del segundo año de vida. Para poder cumplir con esta recomendación, es imprescindible conocer las razones por las cuales las madres abandonan el amamantamiento antes de esa edad y si ciertos grupos de niños están en mayor riesgo de tener una lactancia demasiado corta. 
En un estudio prospectivo de tipo comunitario se siguió a un grupo de 1678 niños de Guinea Bissau desde el nacimiento hasta el cese de la lactancia materna, la emigración o la muerte. La edad mediana de los niños cuando dejaron de mamar fue de 22,6 meses. La presencia de enfermedad materna o infantil o de un nuevo embarazo se asoció con un período de lactancia mucho más corto que cuando el abandono se produjo porque el niño ya estaba "sano" o "lo suficientemente grande". Estas explicaciones mostraron un impacto independiente de otros factores que también influyen en el cese del amamantamiento, como el grupo étnico, la edad materna, la educación de la madre, el orden de los nacimientos y el número de hermanitos muertos. El abandono de la lactancia antes de los 12 meses de edad se asoció exclusivamente con la enfermedad de la madre o del niño y con un nuevo embarazo, pero no con factores socioeconómicos o culturales.

En conclusión, los trabajadores de la salud deben esmerarse por fomentar el amamantamiento cuando la madre o el niño se encuentran enfermos. En vista de que el abandono prematuro de la lactancia a menudo se asocia con la presencia de un embarazo nuevo, la planificación familiar debe formar parte de todo programa para la promoción de la lactancia materna. (Jakobsen MS, et al. Reason for termination of breastfeeding and the length of breastfeeding. Int J Epidemiol 1996;25:115-121).

\title{
Creación de la Sociedad Internacional para la Equidad en Salud
}

Esta Sociedad, denominada International Society for Equity in Health, Inc. (ISEH, Inc.) fue aprobada en 1996 como corporación científica por el estado de Maryland, EUA.

La ISEH tiene como fines: promover la equidad y los servicios de atención a la salud en el ámbito internacional; facilitar el intercambio científico de conocimientos conceptuales y metodológicos en materia de equidad y servicios de salud; fomentar la investigación en el mismo campo, y mantener relaciones con otros organismos regionales e internacionales de intereses afines.

Se espera finalizar la constitución de la ISEH en 1997.

\author{
Información: \\ Dr. José Paganini \\ Grosvenor Park Condominium III \\ 10401 Grosvenor Palace \#807 \\ Rockville, MD 20852
}

